\title{
EXTENSIONS, CROSSED MODULES AND INTERNAL CATEGORIES IN CATEGORIES OF GROUPS WITH OPERATIONS
}

\author{
by T. PORTER
}

(Received 19th September 1985)

In [6] Brown and Spencer noted that internal categories within the category of groups are equivalent to crossed modules. As they remarked, this result was known to various others before them, but it had not until then appeared in print. That paper led me to investigate the question of which algebraic categories, $\mathbf{C}$, were such that a similar result held i.e. internal categories in $\mathbf{C}$ are equivalent to crossed modules of the appropriate type. The resulting work was written up in 1980 but was not submitted for publication.

Since then several things have happened which suggest that the results of that 1980 research may be of considerably wider importance than I had then thought. Various authors have used the group theoretic case in algebraic topology and have generalised it to higher dimensions. Loday [15] has introduced cat ${ }^{n}$-groups which are equivalent to internal $n$-fold categories in groups, and also has given a notion of crossed square corresponding to the case $n=2$. Ellis in his thesis [8] generalised Loday's ideas to arbitrary dimensions and gives this in his paper with Steiner [9]. In fact Ellis proves more as he extends the results of my 1980 preprint to arbitrary dimensions (his Proposition 1.3 .11 p. 30 of [8]). Thus it seems reasonable to publish that earlier work which is the basis for this generalisation.

It is natural however to ask why such an equivalence of categories may be important. Homotopical algebra extends ideas from homotopy theory to algebraic contexts in much the same way as homological algebra extended ideas from classical geometric homology theory. Use of crossed modules of groups has resulted not only in advances in homotopy theory such as the generalised van Kampen theorems of Brown, Higgins and Loday $([2,3,4])$ but, via this theory, to information on the homotopical and homological algebra of groups (again see [2]). Ellis gives purely algebraic proofs of these latter results and is thus able to extend them to apply to Lie algebras. In this context I should also mention the work of Kassell and Loday [13].

Thus crossed modules in other contexts than groups raise the possibility of applying homotopical methods to algebraic problems. The work of Dedecker [7], Gerstenhaber [10] and [11], Lue [17] and [18], and others has already contributed much in this direction, but there is still a lot more to investigate.

However, in any given context, there remains, of course, the initial problem of deciding what a crossed module should be. The test should be a form of the BrownSpencer result. 
As with the original result of Brown and Spencer, no proofs of the equivalence of categories given below has previously been published, but the result is known to various people who discovered it about the same time as me and for the same reasons, i.e. the wish to be able to apply crossed module techniques in contexts other than groups.

Thus the cases of associative rings and algebras, Lie and Jordan algebras are mentioned in Lavendhomme and Roisin [14]. They also give an example $(C=$ Monoids) in which only a more restricted version of the equivalence holds. A detailed discussion of this latter case is implicit in Porter [22] in which the partially algebraic case of $\mathbf{C}=$ Cat, the category of small categories is studied; another partially algebraic case, $\mathbf{C}=$ Groupoids, is essentially treated in a second paper by Brown-Spencer [5]. Aznar Garcia, in his unpublished thesis [1], discusses in detail the general case of "categories of interest" or categories of groups with operations looked at here and applies it to generalising Dedecker's non-abelian cohomology theory. He also discusses the existence of free crossed modules in these contexts. This is also treated in Porter [21].

\section{Categories of groups with operations}

For the idea behind the definition given below we refer the reader to Higgins [12] and Orzech [20]. Our axioms are adapted from those of Orzech.

Let $\Omega$ be a set of finitary operations such that the following axioms hold: If $\Omega_{i}$ is the set of $i$-ary operations in $\Omega$ :

(1) $\Omega=\Omega_{0} \cup \Omega_{1} \cup \Omega_{2}$.

(2) The group operations of identity, inverse and multiplication (written $0,-,+$ ) are elements of $\Omega_{0}, \Omega_{1}, \Omega_{2}$ respectively.

Let $\Omega_{2}^{\prime}=\Omega_{2} \backslash\{+\}, \Omega_{1}^{\prime}=\Omega_{1} \backslash\{-\}$ and assume that if $* \in \Omega_{2}^{\prime}$ then $*^{\circ}$ defined by $x *^{\circ} y=y * x$ is also in $\Omega_{2}^{\prime}$. Also assume that $\Omega_{0}=\{0\}$.

(3) If $* \in \Omega_{2}^{\prime}$, then $a *(b+c)=a * b+a * c$.

(4) If $\omega \in \Omega_{1}^{\prime}$, then $\omega$ is a morphism for + and if $* \in \Omega_{2}^{\prime}$ then $\omega(a) * b=\omega(a * b)$.

Remarks. The set, $\Omega_{0}$ contains exactly one element, the group identity; hence for instance associative rings with unit are not groups with operations. Higgins [12] allows non-trivial ternary and higher operations, but we will not work in this generality.

From now on, $\mathbf{C}$ will denote a category of $\langle\Omega, E\rangle$-algebras where $E$ is a set of identities including the group laws (cf. MacLane [19]). The objects of $C$ may be referred to as groups with operations. The elementary properties of $\mathbf{C}$ can be found in Orzech [20].

\section{Structures and crossed modules}

Let $B, A$ be objects of $C$. An extension of $B$ by $A$ is a sequence

$$
0 \rightarrow A \stackrel{i}{\rightarrow} E \stackrel{p}{\rightarrow} B \rightarrow 0
$$

in which $p$ is surjective and $i$ is the kernel of $p$. 
EXTENSIONS, CROSSED MODULES AND INTERNAL CATEGORIES 375

An object $A$ is singular if it is abelian as a group and if $a_{1} * a_{2}=0$ for all $a_{1}, a_{2} \in A, * \in \Omega_{2}^{\prime}$.

An extension

$$
0 \rightarrow A \stackrel{i}{\rightarrow} E \stackrel{p}{\rightarrow} B \rightarrow 0
$$

is singular if $A$ is singular and is split if there is a morphism $s: B \rightarrow E$ such that $p s=i d_{B}$. A split extension of $B$ by $A$ is called a $B$-structure on $A$.

Given a $B$-structure on $A$

$$
0 \rightarrow A \rightarrow E \underset{s}{\stackrel{p}{\rightleftarrows}} B \rightarrow 0
$$

we get actions of $B$ on $A$ corresponding to the operations in $C$ : For any $b \in B, a \in A, * \in \Omega_{2}^{\prime}$ we have as "definition" of these actions

$$
\begin{aligned}
& b \cdot a=s(b)+a-s(b) \\
& b * a=s(b) * a
\end{aligned}
$$

These actions are called derived actions by Orzech [20].

Proposition 1 (Orzech [20]). A set of actions of $B$ on $A$ (one for each operation in $\Omega_{2}$ ) is a set of derived actions if and only if the semi-direct product $B \tilde{\times} A$, that is the $\Omega$-algebra with underlying set $B \times A$ and operations

$$
\begin{aligned}
& (b, a)+\left(b^{\prime}, a^{\prime}\right)=\left(b+b^{\prime},\left(b^{\prime} \cdot a\right)+a^{\prime}\right) \\
& (b, a) *\left(b^{\prime}, a^{\prime}\right)=\left(b * b^{\prime}, b * a^{\prime}+a * b^{\prime}+a * a^{\prime}\right),
\end{aligned}
$$

is an object in $\mathbf{C}$.

For example, for any $B$ in $C$, the obvious action of $B$ on itself corresponding to the split extension

$$
0 \rightarrow B \stackrel{i_{B^{\prime}}}{\rightarrow} B \times \underset{\Delta_{B}}{\stackrel{P_{B}}{\rightleftarrows}} B \rightarrow 0
$$

also corresponds to the extension

$$
0 \rightarrow B \stackrel{i}{\rightarrow} B \tilde{\times} B \underset{s}{\stackrel{P}{\rightleftarrows}} B \rightarrow 0
$$

with $s(b)=(b, 0)$. 
In the usual way one can talk of morphisms of extensions and morphisms of split extensions. If there is a $B$-structure on $A$ and a $B^{\prime}$-structure on $A^{\prime}$, a pair of morphisms $(\beta, \alpha)$, with $\beta: B \rightarrow B^{\prime}, \alpha: A \rightarrow A^{\prime}$, is a morphism of structures if it gives a morphism of the corresponding split extensions. This happens if and only if for all $a \in A, b \in B, * \in \Omega_{2}^{\prime}$ one has

$$
\begin{gathered}
\alpha((-b) \cdot a)=(-\beta(b)) \cdot \alpha(a) \\
\alpha(b * a)=\beta(b) * \alpha(a) .
\end{gathered}
$$

Given a $B$-structure on $A$ and a morphism (in C) $\phi: A \rightarrow B$, we say $\phi$ is a crossed module if $\left(1_{B}, \phi\right)$ and $\left(\phi, 1_{A}\right)$ are both morphisms of structures.

So as to be able to link this concept with the classical ones-crossed module, crossed ring etc. it is useful to have a description in terms of actions and operations.

Proposition 2. Given a $B$-structure on $A, \phi: A \rightarrow B$ is a crossed module if and only if the following conditions are satisfied for all $a, a_{1}, a_{2} \in A, b \in B, * \in \Omega_{2}^{\prime}$

(i) $\phi((-b) \cdot a)=-b+\phi(a)+b$

(ii) $\left(-\phi\left(a_{1}\right)\right) \cdot a_{2}=-a_{1}+a_{2}+a_{1}$

(iii) $\phi\left(a_{1}\right) * a_{2}=a_{1} * a_{2}=a_{1} * \phi\left(a_{2}\right)$

(iv) $\left\{\begin{array}{l}\phi(b * a)=b * \phi(a) \\ \phi(a * b)=\phi(a) * b .\end{array}\right.$

The proof is straightforward.

As a corollary one finds that when $\mathbf{C}=$ Groups, the crossed modules are exactly the crossed modules of Whitehead [24].

Proposition 3. If $\phi: A \rightarrow B$ is a crossed $(B-)$ module then Ker $\phi$ is singular.

Although the proof is easy we give it as it illustrates how one works with these properties.

Suppose $a_{1}, a_{2} \in \operatorname{Ker} \phi$, then by (ii) above

$$
-a_{1}+a_{2}+a_{1}=\left(-\phi\left(a_{1}\right)\right) \cdot a_{2}=a_{2} \text {. }
$$

So $\operatorname{Ker} \phi$ is an abelian group under + . By (iii) we have

$$
a_{1} * a_{2}=\phi\left(a_{1}\right) * a_{2}=0 * a_{1}=0
$$

Note singular objects are internal groups in $C$.

\section{The equivalence}

An internal category in $\mathbf{C}$ is a diagram in $\mathbf{C}$ 


$$
C \underset{s}{\stackrel{\substack{d_{0} \\ d_{1}}}{\rightarrow}} B
$$

such that $d_{0} s=d_{1} s=i d_{B}$ with an operation $\circ: C \times{ }_{B} C \rightarrow C$ satisfying the usual axioms of a category.

If, in addition, for any $c \in C$, there is $c^{\prime} \in C$ with $c \circ c^{\prime}=s d_{0}(c), c^{\prime} \circ c=s d_{1}(c)$ then we say the category is a groupoid. Note that since $s$ is in $\mathbf{C}, s(0)=0$ and that the operation o being a morphism implies that for all $a, b, c, d \in C, * \in \Omega_{2},(a * b) \circ(c * d)=(a \circ c) *(b \circ d)$ whenever one side makes sense. These are called the interchange laws.

As an easy application we note that any internal category in $\mathbf{C}$ is an internal groupoid, since given $c \in C, c^{-1}=s d_{1}(c)-c+s d_{0}(c)$ satisfies $c^{-1} \circ c=s d_{1}(c), c \circ c^{-1}=s d_{0} c$.

For future use we note the following two lemmas.

Lemma A. Given $c_{1}, c_{2} \in C$ with $c_{1} \in \operatorname{Ker} d_{0}, c_{2} \in \operatorname{Ker} d_{1}$, then for any $* \in \Omega_{2}^{\prime}$

$$
c_{2}+c_{1}=c_{1}+c_{2}
$$

and

$$
c_{2} * c_{1}=c_{1} * c_{2}=0 \text {. }
$$

The proof is an easy use of the interchange laws.

Lemma B. If $c^{\prime} \in \operatorname{Ker} d_{0}$ then, for any $* \in \Omega_{2}^{\prime}$, we have, for all $c \in C$,

$$
\left(-s d_{1}(c)\right) \cdot c^{\prime}=-c+c^{\prime}+c
$$

and

$$
c^{\prime} * s d_{1}(c)=c^{\prime} * c, \quad s d_{1}(c) * c^{\prime}=c * c^{\prime}
$$

Proof. As $c^{\prime} \in \operatorname{Ker} d_{0}$ and $c-s d_{1}(c) \in \operatorname{Ker} d_{1}$, we can apply Lemma $A$. The two cases are then easy consequences of the corresponding parts of that lemma.

We can now associate to a category in $\mathbf{C}$, a crossed module $\phi$ as follows: Given

$$
C \underset{\frac{d_{1}}{\stackrel{d_{1}}{\longrightarrow}}}{\stackrel{d_{0}}{\longrightarrow}} B
$$


set $A=\operatorname{Ker} d_{0}, \phi=d_{1} \mid A$. Certainly

$$
0 \rightarrow \mathrm{A} \rightarrow \mathrm{C} \underset{s}{\stackrel{d_{0}}{\rightleftarrows}} \mathrm{B} \rightarrow 0
$$

gives a $B$-structure on $A$. We have:

Proposition 4. With the above notation $\phi: A \rightarrow B$ is a crossed module.

In fact criteria (i) and (iv) are trivial consequences of the way the $B$-structure on $A$ is defined and (ii) and (iii) follow from Lemma $B$.

Thus we have functors

$$
\phi: \operatorname{Cat}(\mathbf{C}) \rightarrow X \operatorname{Mod}(\mathbf{C})
$$

and for each $B$ in $\mathbf{C}$

$$
\phi: \operatorname{Cat}_{B}(\mathbf{C}) \rightarrow X B-\operatorname{Mod}(\mathbf{C})
$$

where $\mathrm{Cat}_{B}(\mathbf{C})$ is the category of internal categories in $\mathbf{C}$ with objects $B$; the other notation is self explanatory.

Remark. It is an easy consequence of the interchange laws that the internal category composition is completely determined by the group multiplication; explicitly

$$
c \circ c^{\prime}=c-s d_{0}\left(c^{\prime}\right)+c^{\prime}
$$

This can also be derived from the description of the functors involved in the equivalence of categories.

Remark. $\phi$ is a functor fibred over $\mathbf{C}$ in an obvious way.

Theorem. $\phi$ and $\phi_{B}$ are both equivalences of categories.

We sketch the construction of the quasi-inverses of $\phi$ and $\phi_{B}$ leaving the detailed verification to the reader.

Given a $B$-crossed module $\phi: A \rightarrow B$, put $C=B \tilde{\times} A$ and consider the diagram

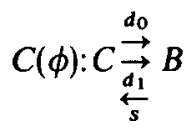

where $s(b)=(b, 0), d_{0}(b, a)=b$ and $d_{1}(b, a)=b+\phi(a)$. To define composition note that $(b, a) \circ(d, c)$ is defined only if $d=b+\phi(a)$ and hence the definition 


$$
(b, a) \circ(b+\phi(a), c)=(b, a+c)
$$

makes sense. Both $d_{1}$ and $\circ$ are morphisms and the verification is fairly straightforward that the resulting structure is an internal category in $\mathbf{C}$.

The assignment $\phi \rightarrow C(\phi)$ is clearly functorial and is the required quasi-inverse for $\phi$ $\left(\right.$ or $\left.\phi_{B}\right)$.

Remarks. (a) A close inspection of the proofs would reveal that all the axioms on $\Omega$ were used at least once; thus it is difficult to see how one might weaken these axioms to get a wider class of algebraic categories in which the result is true.

(b) A special case of the above result occurs when the internal category is a monoid (i.e. has only one object) then we have a proof of the well known result that an internal monoid in $C$ is precisely a singular object.

(c) It is worth noting that associativity of the internal category composition is not used in the proof of the equivalence.

Example. One of the simplest examples of the above is when $C$ is a category of commutative algebras over some commutative ring, $k$, with unit. The algebras in $C$ are not necessarily unitary and if an algebra has a unit, 1 , it is not assumed to be preserved by the morphisms in $C$.

An internal category in commutative $k$-algebras is a diagram

$$
C \underset{s}{\stackrel{d_{0}}{\rightarrow}} B
$$

of $k$-algebra morphisms, together with a composition which we can express in terms of addition by

$$
c \circ c^{\prime}=c-s d_{0}\left(c^{\prime}\right)+c^{\prime}
$$

The interchange laws reduce to one only corresponding to multiplication (c.f. equation (8) p. 255 of [21]). This is still quite complicated however, and it is much easier to describe $C$ in the form $B \times \bar{x}$ where $A=\operatorname{Ker} d_{0}$, that is in the form coming from the equivalence of categories.

In describing the structure of $A$, one notes that the only non-trivial action of $B$ on $A$ is multiplicative and this gives a $B$-module structure to $A$ in such a way that multiplication in $A$ is $B$-bilinear. Finally $\phi=d_{1} \mid A$ satisfies the two crossed module conditions:

(a) $\phi$ is $B$-linear

(b) $\phi(a) a^{\prime}=a a^{\prime}$ for all $a, a^{\prime} \in A$. 
Related structures have been studied by Gerstenhaber [10], Lichtenbaum and Schlessinger [16], Lue [18] and others. A more recent application is to be found in [23].

\section{REFERENCES}

1. E. R. Aznar Garcia, Cohomologia no abeliana en categories de intres (Ph.D. Thesis, Univ. Santiago de Compostela, 1981), available as Alxebra 33.

2. R. Brown, Some non-abelian methods in homotopy theory and homological algebra, Proc. Conf. categorial topology, Toledo, Ohio, 1983 (Heldermann Verlag 1984).

3. R. Brown and P. J. Higgins, Colimits of relative homotopy groups, J. Pure Appl. Algebra 22 (1981), 11-41.

4. R. Brown and J.-L. Loday, van Kampen theorems for diagrams of spaces, I, U.C.N.W., Pure Maths. Preprint 84.7, Topology, to appear.

5. R. Brown and C. B. Spencer, Double groupoids and crossed modules, Cahiers Top. Geom. Diff. 17 (1976), 343-362.

6. R. Brown and C. B. Spencer, G-groupoids, crossed modules and the fundamental group of a topological group, Proc. Kon. Ned. Akad. v. Wetensch. 79 (1976), 296-302.

7. P. Dedecker, Cohomologie non abélienne (Lille, 1965), mimeographed 78 pp.

8. G. J. Elus, Crossed Modules and their Higher Dimensional Analogues (University of Wales Ph.D. Thesis, August, 1984).

9. G. J. Ellis and R. Steiner, Higher dimensional crossed modules and the homotopy groups of $(n+1)-a d s$, U.C.N.W., Pure Maths. Preprint 85.6., 9 pp.

10. M. Gerstenhaber, On the deformation of rings and algebras, Ann. of Math.84 (1966), 1-19.

11. M. Gerstenhaber, A uniform cohomology theory for algebras, Proc. Nat. Acad. Sci. U.S.A. 51 (1964), 624-629. 416.

12. P. J. Higgins, Groups with multiple operators, Proc. London Math. Soc. (3) 6 (1956), 366-

13. C. Kassell and J.-L. Loday, Extensions centrales d'algebres de Lie (Preprint 175, P-99, I.R.M.A., Strasbourg, 1982).

14. R. Lavendhomme and J. Roisin, Cohomologie non-abélienne de structures algébriques, $J$. Algebra 67 (1980), 355-414.

15. J.-L. Loday, Spaces with finitely many homotopy groups, J. Pure Appl. Algebra 24 (1982), 179-202.

16. S. Lichtenbaum and M. Schlessinger, The cotangent complex of a morphism, Trans. Amer. Math. Soc. 128 (1867), 41-70.

17. A. S.-T. Lue, Non-abelian cohomology of associative algebras, Quart. J. Math. Oxford (2) 19 (1968), 159-180.

18. A. S.-T. LuE, Cohomology of algebras relative to a variety, Math. Z. 121 (1971), 220-232.

19. S. MacLane, Categories for the Working Mathematician (Graduate texts in Maths Vol. 5, Springer, 1971).

20. G. ORZECH, Obstruction theory in algebraic categories I and II, J. Pure Appl. Algebra 2 (1972) 287-314 and 315-340. 
21. T. Porter, Internal Categories and Crossed Modules, in Category Theory, Proceedings, Gummersbach 1981 (Lecture Notes in Mathematics Vol. 962, 1982, Springer-Verlag), 245-255.

22. T. Porter, Crossed modules in Cat and a Brown-Spencer Theorem for 2-categories, Cahier. Top. Géom. Diff. Catégories 26 (1985), 381-388.

23. T. PORTER, Homology of commutative algebras and an invariant of Simis and Vasconcelos, J. Algebra 99 (1986), 458-465.

24. J. H. C. WhItehead, Combinatorial homotopy II, Bull. Amer. Math. Soc. 55 (1949), 453-496.

Department of Pure Mathematics

University College of North Wales

BANGOR

GWYNEDD LL57 2UW

WALES 